100:8466-8471.

29. Mootha, V., et al. 2003. PGC-1alpha responsive genes involved in oxidative phosphorylation are coordinately downregulated in human diabetes. Nat. Genet. 34:267-273.

30. Stump, C., et al. 2003. Effect of insulin on human skeletal muscle mitochondrial ATP production, protein synthesis and mRNA transcripts. Proc. Natl. Acad. Sci. U. S. A. 100:7996-8001.

31. Kelley, D.E., et al. 2002. Dysfunction of mitochondria in human skeletal muscle in type 2 diabetes.
Diabetes. 51:2944-2950.

32. Ritov, V., et al. 2005. Deficiency of sub-sarcolemmal mitochondria in obesity and type 2 diabetes. Diabetes. 55:8-14.

33. Wisloff, U., et al. 2005. Cardiovascular risk factors emerge after artificial selection for low aerobic capacity. Science. 307:418-420.

34. Simoneau, J., and Kelley, D. 1997. Altered glycolytic and oxidative capacities of skeletal muscle contribute to insulin resistance in NIDDM. J. Appl. Physiol. 83:166-171.
35. Simoneau, J.-A., et al. 1999. Markers of capacity to utilize fatty acids in human skeletal muscle: relation to insulin resistance and obesity and effects of weight loss. FASEB J. 13:2051-2060.

36. Zurlo, F., et al. 1994. Whole body energy metabolism and skeletal muscle biochemical characteristics. Metabolism. 43:481-486.

37. Menshikova, E., et al. 2005. Effects of weight loss and physical activity on skeletal muscle mitochondrial function in obesity. Am. J. Physiol. Endocrinol. Metab. 288:E818-E825.

\title{
HIF-1 $\alpha$ : a master regulator of innate host defenses?
}

\author{
Kol A. Zarember and Harry L. Malech
}

Laboratory of Host Defenses, National Institute of Allergy and Infectious Diseases, National Institutes of Health, Bethesda, Maryland, USA.

In the days following infection, when the human body develops and refines antibodies and prepares to mount an adaptive immune response, the bulwark of innate host defense against microbial infection is the polymorphonuclear leukocyte (PMN). PMNs seek out, identify, engulf, and sterilize invading microbes using both $\mathrm{O}_{2}$-dependent and $\mathrm{O}_{2}$-independent antimicrobial systems. A decrease in PMN numbers or function caused by immunosuppression or disease increases the risk of infection. In this issue of the JCI, Peyssonnaux et al. identify a novel and essential role for hypoxia-inducible factor- $1 \alpha$ in regulating several important PMN functions relevant to host defense, including transcription of cationic antimicrobial polypeptides and induction of NO synthase (see the related article beginning on page 1806).

Hypoxia-inducible factor 1 (HIF-1) is a multisubunit protein that regulates transcription at hypoxia response elements (HREs) and is composed of 2 basic helix-loop-helix proteins: the $\alpha$ subunit, HIF- $1 \alpha$, and the constitutively expressed HIF- $1 \beta$ (also known as aryl hydrocarbon receptor nuclear translocator [ARNT]). As depicted in Figure 1A, during normoxia HIF- $1 \alpha$ is hydroxylated on several proline and asparaginyl residues, which enables high-affinity binding of HIF- $1 \alpha$ to von Hippel-Lindau tumor suppressor protein (vHL), a component of a ubiquitin ligase complex that ubiquitinates and thereby targets HIF- $1 \alpha$ for proteosomal degradation. Under hypoxic conditions the

Nonstandard abbreviations used: CEBP, CCAAT enhancer-binding protein; CRAMP, cathelicidin-related antimicrobial peptide; FIH, factor inhibiting HIF-1 $\alpha$; HIF-1, hypoxia-inducible factor 1 ; HRE, hypoxic response element; PMN, polymorphonuclear leukocyte; vHL, von Hippel-Lindau tumor suppressor protein.

Conflict of interest: The authors have declared that no conflict of interest exists.

Citation for this article: J. Clin. Invest. 115:1702-1704 (2005). doi:10.1172/JCI25740.
$\mathrm{O}_{2}$-dependent hydroxylation of HIF- $1 \alpha$ is decreased, which prevents its degradation (Figure 1B). A further level of $\mathrm{O}_{2}$-dependent regulation exists: the hydroxylation of an asparagine residue by factor inhibiting HIF-1 $\alpha$ (FIH) blocks the interaction of HIF- $1 \alpha$ with p300/CBP transcriptional coactivator proteins, thereby decreasing transcription of HIF- $1 \alpha$-regulated genes at normoxia. When HIF-1 $\alpha$ levels increase in response to hypoxia in tissues (or after stimulation of myeloid cells with bacteria, as discussed below), functional HIF-1 regulates transcription at HREs of target gene regulatory sequences, which results in the transcription of genes such as Erythropoietin and VEGF and thereby enhances local vascularization and systemic oxygen transport (Figure 1B). The details of the regulation of the general hypoxia response have been thoroughly discussed in several reviews (1-3).

\section{A role for HIF-1 $\alpha$ in myeloid cells}

The knockout of HIF- $1 \alpha$ in mice is embryonically lethal due to its essential role in the development of the vascula- ture. To circumvent this lethality and allow further examination of the role of HIF- $1 \alpha$ in phagocytes, Cramer et al. employed a myeloid cell-specific HIF- $1 \alpha-$ knockout approach and found that HIF- $1 \alpha$ was an important regulator of myeloid cell metabolism (by decreasing cellular ATP levels), neutrophil bactericidal potency, and macrophage migration (4). In this issue of the JCI, Peyssonnaux et al. (5) built on this foundation and attempted to understand the specific molecular defects contributing to depressed myeloid cell function in the absence of HIF- $1 \alpha$. Temporarily leaving aside the consequences of the absence of HIF-1 $\alpha$, the authors also discovered that in addition to being activated by hypoxia, HIF-1 $\alpha$ was equivalently upregulated in WT macrophages at normoxia following exposure to Gram-positive (Streptococcus and Staphylococcus) and Gram-negative (Salmonella and Pseudomonas) bacteria. HIF-1 $\alpha$ was previously shown to be activated in macrophages treated with LPS (6), a microbial activator of TLR-4. The 10 human TLRs are the cellular sentinels of microbial recognition. They respond to a variety of microbial products (e.g., LPS, lipoproteins, proteins, and nucleic acids) by activating signaling pathways leading to NF-кB-mediated transcriptional regulation and, in some cases, activation of Rac1 and PI3K, which may regulate more rapid cellular responses (7). Regardless of the exact position along their signal cascades at which the TLR and HIF-1 $\alpha$ pathways intersect, these observations illuminate a fertile territory for further study. To what extent, if any, do the transcrip- 


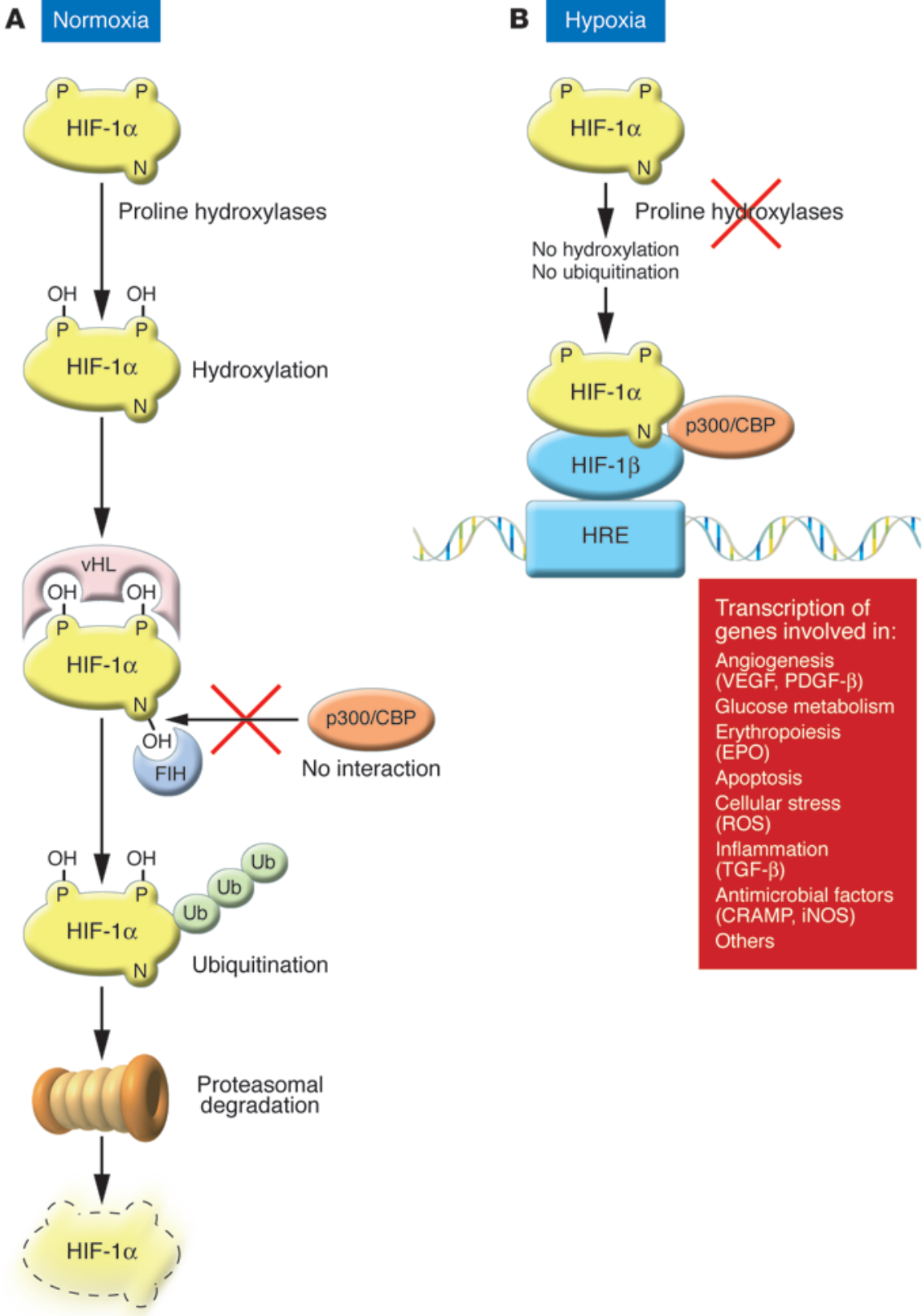

Figure 1

HIF- $1 \alpha$ regulates several important PMN functions relevant to host defense during both normoxia and hypoxia. (A) During normoxia, $\mathrm{O}_{2}$-dependent proline hydroxylases modify HIF-1 $\alpha$ proline residues 402 and 564. Asparagine 803 is hydroxylated by $\mathrm{FIH}$, which decreases HIF-1 $\alpha$ interaction with the $\mathrm{p} 300 / \mathrm{CBP}$ transcriptional coactivators. The hydroxylated prolines are recognized by $\mathrm{vHL}$, a component of a ubiquitin ligase complex that ubiquitinates (Ub) HIF-1 $\alpha$ and thereby targets it for proteosomal degradation. (B) During hypoxia and/or bacterial infection, when proline hydroxylases are not active, HIF-1 $\alpha$ regulates transcription at HREs by accumulating and binding to HIF-1 $\beta$ and $\mathrm{p} 300 / \mathrm{CBP}$, which results in transcription of hypoxia-inducible genes involved in angiogenesis, glucose transport and metabolism, erythropoiesis, inflammation, apoptosis, and cellular stress. EPO, erythropoietin.

tional programs regulated by HIF-1 and NF- $\kappa \mathrm{B}$ overlap and differ? Do all TLRs activate HIF-1 to the same extent?

In contrast to the findings of Cramer et al. (4), in which the recruitment of HIF-1 $\alpha^{-/-}$ polymorphonuclear lymphocytes (PMNs) to affected tissues was found to be defective in mice after exposure to a chemical irritant, Peyssonnaux et al. (5) found that HIF-1 $\alpha^{-/-}$PMNs were recruited to sites of microbial infection in vivo as efficiently as were WT PMNs and were also equally able to generate ROS (a PMN microbicide). Interestingly, iNOS mRNA is induced by bacteria in a HIF-1 $\alpha$-dependent fashion and nitrite production (a measure of $\mathrm{NO}$ production) is decreased in HIF- $1 \alpha^{-/-}$ cells. NO itself acts as a microbicide, but the authors demonstrate that it was involved in regulating the expression of HIF-1 $\alpha$. Further, both in vivo and in vitro, HIF-1 $\alpha^{-/-}$PMNs were less able to kill Gram-positive and -negative bacteria. This observation prompted Peyssonnaux et al. to examine the activity of one of the many known $\mathrm{O}_{2}$-independent antimicrobial proteins, cathelicidin-related antimicrobial peptide (CRAMP). Cathelicidins were initially thought to be stored in PMNs as inactive proforms that required the actions of elastase, proteinase 3, or gastricsin to release a cationic C-terminal peptide with antimicrobial activity (8). However, recent studies have shown potentially important innate defense activities for unprocessed cathelicidins (e.g., LPS neutralization and chemoattractant activity). The results of Peyssonnaux et al. indicate that HIF- $1 \alpha$ is apparently required for the synthesis of CRAMP mRNA and protein, and deletion of vHL causes increased expression of CRAMP mRNA (Figure 1B). The cathelicidin-activating protease neutrophil elastase was found to be similarly regulated. Not only would reduced elastase activity decrease cathelicidin processing and antimicrobial activity (9), but elastase itself is directly antimicrobial (10). While HIF- $1 \alpha$ regulates cathelicidin expression, it has also been reported that a pig cathelicidin peptide, PR-39, can itself induce angiogenesis by inhibiting the degradation of HIF- $1 \alpha$ and subsequent induction of VEGF (11). The human cathelicidinderived peptide LL-37 promotes angiogenesis in a VEGF-independent manner, and cathelicidin-deficient mice have impaired angiogenesis (12). If human LL-37 also upregulates HIF- $1 \alpha$ activity, its processing would promote further synthesis of cathelicidin, elastase, NO, and other HIF- $1 \alpha-$ regulated gene products as well as vascularization during wound repair.

In general, the pleiotropic effects of global regulators can complicate the dissociation of their primary (direct) effects from their secondary effects. No results of microarray analysis of gene expression profiles of HIF-1 $\alpha^{-/-}$myeloid cells have yet been reported; these may help identify 
HIF- $1 \alpha$-dependent transcription factors whose absence could be directly responsible for some of the observed defects in HIF- $1 \alpha$ KO PMNs. For example, it has been shown that mutations in the myeloid transcription factor CCAAT enhancerbinding protein $-\varepsilon$ (CEBP- $\varepsilon$ ) can lead to secondary granule deficiency, a genetic disorder causing impaired bactericidal capacity in human PMNs and increased risk of infection (13), and are associated with decreased expression of antimicrobial proteins such as CRAMP (14).

\section{vHL and immunity}

Given the regulatory role of vHL in degrading HIF-1 $\alpha$ during normoxia, one would expect the absence of functional vHL to increase HIF- $1 \alpha$ levels and HIFdependent transcripts. Peyssonnaux et al. (5) used vHL KO mice to confirm this prediction. It will be of interest to examine the PMNs of patients with von HippelLindau disease in which the VHL gene is lost and test whether these cells are better able to kill microbes as suggested by the increased expression of cathelicidins and elastase observed in vHL-null neutrophils by Peyssonnaux et al. (5). Different mutations in vHL are associated with Chuvash polycythemia, a hypoxia-sensing disorder characterized by homozygous mutation of VHL. These patients exhibit increased erythropoietin and VEGF expression but they do not appear to suffer from increased tumorigenesis associated with von Hippel-Lindau disease (15), which suggests that additional signals emanate from $v H L$ that may play important roles in its function as well as indirect roles in the function of HIF-1.

\section{HIF-1 $\alpha$ and PMN longevity}

The PMN is a relatively short-lived cell (its half-life in the circulation is about 6 hours) and it exits the bone marrow replete with antimicrobial factors stored in cytoplasmic granules. The few ribosomes and scant ER possessed by mature PMNs has led to the general impression that the biosynthetic capacity of the PMN is limited. However, the PMN's lifespan is increased significantly by inflammatory stimuli such as LPS and IL-1 (16). In addition, it was recently shown that hypoxia actually delays apoptosis of PMNs in a HIF-1 $\alpha$-dependent fashion $(17,18)$, so that the same signals that induce the expression of CRAMP mRNA
- and other HIF-1 $\alpha$-dependent proteins expressed by PMNs - would also allow the PMN to survive (6) and perhaps allow sufficient time for the limited biosynthetic capacity of these cells to contribute functionally to innate immune defense.

PMNs accumulate at sites of inflammation or infection and when activated they release antimicrobial factors, including ROS such as $\mathrm{H}_{2} \mathrm{O}_{2}$ and $\mathrm{HOCl}$ (the active ingredient in household bleach), which promote the killing of extracellular microbes. ROS are generated by NADPH oxidase systems in PMNs in a process referred to as the respiratory burst. Peyssonnaux et al. (5) found that HIF-1 $\alpha^{-/-}$ PMNs generated normal levels of ROS. It has been previously recognized, and is also shown in the current study, that inflammatory loci become hypoxic in vivo. Indeed the respiratory burst of PMNs was first recognized by a decrease in $\mathrm{O}_{2}$ levels measured using a Warburg manometer (19). It may well be that HIF-1 $\alpha$ is activated in vivo by this depression in local $\mathrm{O}_{2}$ (in addition to bacteria- and TLR agonist-induced activation), which leads to increased synthesis of $\mathrm{O}_{2}$-independent antimicrobial polypeptides (such as cathelicidins) by PMNs. It will be very interesting to see if patients with chronic granulomatous disease, whose myeloid cells lack the enzymatic machinery required to generate a respiratory burst, are consequently unable to generate a second signal that would normally lead to HIF- $1 \alpha-$ mediated induction of $\mathrm{O}_{2}$-independent antimicrobial systems.

By dissecting the role of HIF- $1 \alpha$ in innate immune defenses, the study by Peyssonnaux et al. (5) introduces new targets for therapeutic immunomodulation. Several compounds found to activate HIF- $1 \alpha$ in vitro have been used in the clinic for other purposes and appear to be well tolerated (e.g., mimosine, $\mathrm{CoCl}_{2}$, and desferrioxamine). These compounds may increase the production of cationic antimicrobial polypeptides and $\mathrm{NO}$ through activation of HIF- $1 \alpha$ and thereby augment the production of endogenous antibiotics. The complex web of associations of HIF- $1 \alpha$ with the TLR signaling pathway and with vHL may provide abundant opportunities for therapeutic intervention in immune and inflammatory disorders, cancer, and vascular development. Further studies of the ways in which HIF- $1 \alpha$ orchestrates effective innate immune defenses are therefore urgently needed.

Address correspondence to: Harry L. Malech, Laboratory of Host Defenses, National Institute of Allergy and Infectious Diseases, National Institutes of Health, 10 Center Drive, MSC 1456, Bethesda, Maryland 20892-1456, USA. Phone: (301) 402-1802; Fax: (301) 4020789; E-mail: HMALECH@niaid.nih.gov.

1. Semenza, G.L. 2004. Hydroxylation of HIF-1: oxygen sensing at the molecular level [review]. Physiology (Bethesda). 19:176-182.

2. Semenza, G.L. 2000. HIF-1 and human disease: one highly involved factor [review]. Genes Dev. 14:1983-1991.

3. Bruick, R.K. 2003. Oxygen sensing in the hypoxic response pathway: regulation of the hypoxiainducible transcription factor [review]. Genes Dev. 17:2614-2623.

4. Cramer, T., et al. 2003. HIF-1alpha is essential for myeloid cell-mediated inflammation [erratum 2003, 113:419]. Cell. 112:645-657.

5. Peyssonnaux, C., et al. 2005. HIF-1 $\alpha$ expression regulates the bactericidal capacity of phagocytes. J. Clin. Invest. 115:1806-1815. doi:10.1172/JCI23865. 6. Blouin, C.C., Page, E.L., Soucy, G.M., and Richard, D.E. 2004. Hypoxic gene activation by lipopolysaccharide in macrophages: implication of hypoxiainducible factor 1alpha. Blood. 103:1124-1130.

7. Arbibe, L., et al. 2000. Toll-like receptor 2-mediated NF-kappa B activation requires a Rac1-dependent pathway. Nat. Immunol. 1:533-540.

8. Zanetti, M. 2004. Cathelicidins, multifunctional peptides of the innate immunity. J. Lenkoc. Biol. 75:39-48.

9. Cole, A.M., et al. 2001. Inhibition of neutrophil elastase prevents cathelicidin activation and impairs clearance of bacteria from wounds. Blood. 97:297-304.

10. Belaaouaj, A., et al. 1998. Mice lacking neutrophil elastase reveal impaired host defense against gram negative bacterial sepsis. Nat. Med. 4:615-618.

11. Li, J., et al. 2000. PR39, a peptide regulator of angiogenesis [erratum 2000, 6:356]. Nat. Med. 6:49-55.

12. Koczulla, R., et al. 2003. An angiogenic role for the human peptide antibiotic LL-37/hCAP-18. J. Clin. Invest. 111:1665-1672. doi:10.1172/JCI200317545.

13. Lekstrom-Himes, J.A., Dorman, S.E., Kopar, P., Holland, S.M., and Gallin, J.I. 1999. Neutrophilspecific granule deficiency results from a novel mutation with loss of function of the transcription factor CCAAT/enhancer binding protein epsilon. J. Exp. Med. 189:1847-1852.

14. Verbeek, W., et al. 1999. Myeloid transcription factor C/EBPepsilon is involved in the positive regulation of lactoferrin gene expression in neutrophils. Blood. 94:3141-3150.

15. Ang, S.O., et al. 2002. Disruption of oxygen homeostasis underlies congenital Chuvash polycythemia. Nat. Genet. 32:614-621.

16. Colotta, F., Re, F., Polentarutti, N., Sozzani, S., and Mantovani, A. 1992. Modulation of granulocyte survival and programmed cell death by cytokines and bacterial products. Blood. 80:2012-2020.

17. Hannah, S., et al. 1995. Hypoxia prolongs neutrophil survival in vitro. FEBS Lett. 372:233-237.

18. Walmsley, S.R., et al. 2005. Hypoxia-induced neutrophil survival is mediated by HIF-1alpha-dependent NF-kappaB activity. J. Exp. Med. 201:105-115.

19. Baldridge, C.W., and Gerard, R.W. 1933. The extra respiration of phagocytosis. Am. J. Physiol. 103:235-236. 